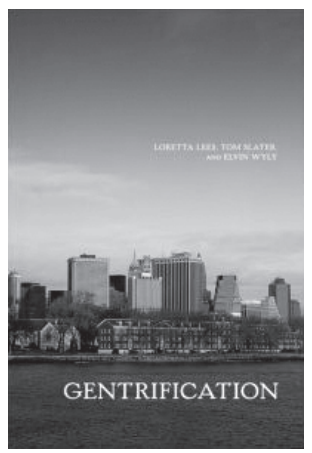

\title{
Loretta Lees, Tom Slater \& Elvin Wyly. Gentrification
}

\author{
London: Routledge, 2008, 310 p.
}

\author{
Ernesto López Morales ${ }^{1}$
}

Los estudios sobre gentrificación, o elitización como ha sido traducido al castellano (García, 2001), parecieran estar experimentando gran surgimiento en el mundo en los últimos años. Sin embargo, la interpretación de sus causas y efectos siguen siendo tema de gran controversia. Esto se debe a que, en primer lugar, la gentrificación es un desafío aún no resuelto para las teorías tradicionales de localización residencial y estructura social urbana. Muy pocos enfoques neoclásicos han explicado, mucho menos modelado, las causas del fenómeno. Segundo, es un desafío tanto para la política como las políticas urbanas, ya que confronta temas cruciales de regeneración y desplazamiento social de áreas centrales y pericentrales urbanas. Tercero, la gentrificación en la actualidad representa uno de los principales campos de batalla teóricos e ideológicos para el urbanismo y la geografía urbana, con posturas epistemológicas y políticas contrastadas. En este contexto, Gentrification viene a compilar y analizar en profundidad la producción internacional más relevante existente en torno a este tema, desde la década de 1960 en adelante, y podría ser incluso una referencia clave para los estudios de la gentrificación en Latinoamérica, en los próximos años.

Tras revisar el nacimiento de la observación científica de la gentrificación en los años sesenta, pasando por las transformaciones de Brooklyn (Nueva York) y los estudios de Glass en Islington (Londres), el libro se

\footnotetext{
1 Departamento de Urbanismo, Universidad de Chile (Chile). E-mail: erlopez@uchile.cl
}

centra en un primer gran tema, como son las contradicciones actuales epistemológicas asociadas al fenómeno y sus dos principales corrientes teóricas. Una primera definición denominada enfoque de la oferta, entiende la gentrificación como producto de una acumulación oligopólica de la renta urbana en contextos de economías de mercado. Neil Smith $(1979,1996)$ es probablemente el autor de mayor contribución en este ámbito. De acuerdo a su teoría del rent gap (o brecha de renta), es clave el diferencial entre una renta futura potencial y una renta actual capitalizada existente en las zonas pericentrales, un valle en la curva de valor de suelo que genera un enorme potencial de beneficio para los desarrolladores inmobiliarios (generadores de oferta). Una expandida brecha de renta definirá el dónde y cuándo existen las mejores condiciones para la acumulación de la renta urbana, lo que se lleva a cabo de la mano de los desarrolladores y propietarios arrendadores a través de acciones concretas orientadas a devaluar al máximo la renta actual capitalizada y apreciar lo más posible la renta potencial. Según esta teoría, la gentrificación de la ciudad es de por sí el producto de una racionalidad económica orientada a la acumulación de esa renta potencial. Si bien este enfoque ha sido comúnmente catalogado como económicodeterminista, los autores del libro opinan que tal crítica es limitante, ya que, por el contrario, la teoría del rent gap aparece hoy como un lente imprescindible para observar regulaciones públicas y conflictos de poder generados alrededor de la distribución desigual de la plusvalía urbana, temas clave en Latinoamérica. El libro revisa en profundidad aportes contemporáneos en esta línea. 
Un enfoque distinto plantea que las causas principales de la gentrificación se hallan en los cambios culturales y de estructura ocupacional, a la manera de las ciudades postindustriales en los países de economías avanzadas. Ley (1980) y Hamnett (1994) son referencias clásicas de este modelo. Contrariamente a la teoría de la oferta, estas explicaciones (basadas en la demanda) otorgan mayor importancia a los cambios en las preferencias de consumo, trabajo y residencia, experimentados por clases acomodadas que buscan diferenciarse de los estilos de vida suburbanos asociables a un régimen anterior de producción fordista-industrial. La tesis aquí implícita es que existiría una verdadera "ideología liberal de la renovación urbana", postmoderna, más basada en la calidad de vida que en el crecimiento económico. Este enfoque ha sido adoptado en el último tiempo por mayor diversidad de autores en el mundo, con distintas interpretaciones, en general porque las transformaciones culturales y de consumo de suelo urbano parecieran ser más evidentes que las variaciones en la renta urbana o los desplazamientos sociales, en tanto causas o efectos de la gentrificación. En cualquier caso, en la actualidad, disputas tan extremas sobre las causas del fenómeno parecieran ser ya excesivas, a la vez que posturas más contemporáneas tienden a encontrar una síntesis, asimismo reenfocando cada vez más los estudios de la gentrificación hacia los efectos socioespaciales producidos, y no solamente sus causas (Slater, 2006).

Un segundo gran tema analizado por el libro son las recientes transformaciones de escala de la gentrificación. Basándose mayormente en evidencia de países desarrollados, se plantea grosso modo que el fenómeno habría transitado por tres fases: una primera etapa de producción esporádica a escala barrial desarrollada hasta fines de la década de los setenta, observada en ciertas ciudades globales, promovida por políticas públicas orientadas a la generación de un clima propicio para los desarrollos inmobiliarios, bajo la premisa de combatir el deterioro urbano. Una segunda fase ocurre en los años ochenta hasta mediados de los noventa, en que la gentrificación pasa a ser parte de estrategias de desarrollo económico mayores, con capital financiero global invo- lucrado. Esta fase es caracterizada por estrechas alianzas público-privadas (entrepreneurialism) y decisivos subsidios públicos a la oferta, en ciudades no necesariamente globales, y con intensos conflictos sociales emanados de desplazamientos.

Finalmente, tras cierta recuperación mundial de la crisis financiera de fines de la década de 1990, una tercera fase de gentrificación comienza a ser observada tanto en el centro como periferias mundiales, con mayor volumen, escala e internacionalización de capital financiero involucrado, instrumentos de crédito más flexibles (tanto a la oferta como la demanda) y más activa participación de los aparatos públicos centrales y locales en su materialización, observándose, además, cierta marginalización de movimientos antigentrificación. Otras variaciones de carácter espacial son también detectadas en esta última etapa, a saber: gentrificación rural, new-build gentrification y más recientemente supergentrificación, que describe la ocupación de los superricos de zonas previamente gentrificadas en ciudades globales. Sin embargo, resulta casi paradójico que las funciones de los Estados nacionales y locales parecen cuestiones apenas explicadas aún por las teorías actualmente existentes en torno a este tema.

El tercer tópico analizado por Gentrification son las contradicciones de orden político del fenómeno, y los enfoques contradictorios sobre sus efectos en la ciudad. En general, numerosos autores defienden la necesidad de promover una gentrificación positiva en áreas urbanas deprimidas, debido a los varios efectos benéficos que esta generaría, a saber: estabilización de zonas urbanas en decadencia y aumento del valor de las propiedades, disminución de vivienda inutilizada, aumento de la base impositiva fiscal local, estímulo a redesarrollos posteriores, reducción de la expansión excesiva de la ciudad, aumento de beneficios emanados de mix sociales y rehabilitación de propiedades deterioradas con o sin apoyo estatal. Sin embargo, también existe concordancia en que los efectos negativos observados en el mundo serían largamente más contundentes, a saber: desplazamiento social por aumentos en los arriendos y valores de propiedades, efectos psicológicos secundarios asociados 
a los desplazamientos (resentimiento y conflicto en las comunidades desplazadas), disminución de vivienda económica disponible en las zonas en renovación, aumento del valor de propiedades debido a especulación inmobiliaria, aumento de población sin techo, alto uso de recursos públicos para promover ciertas áreas, desplazamiento de usos industriales y comerciales y aumento de los costos de los servicios remanentes, presión sobre áreas urbanas contiguas, pérdida de heterogeneidad social en los barrios, disminución de la tasa de ocupación de viviendas y, en general, pérdida de población en áreas gentrificadas (Atkinson y Bridge, 2005).

Este último punto es extremadamente sensible para los autores del libro, quienes discuten el factor de desplazamiento como condición necesaria (casi exclusiva, según algunos autores) para la gentrificación, ya que también existen formas de "desplazamiento por exclusión", uno de cuyos ejemplos más destacables es el creciente número de enclaves cerrados en el mundo (notablemente en las urbes latinoamericanas). De tal forma, la aparente inexistencia de desplazamiento espacial y la proximidad geográfica entre diferentes clases sociales como condiciones suficientes para una gentrificación positiva, resultarían ser factores bastante discutibles. Investigaciones más recientes muestran también que procesos de revanchismo social y los limitados resultados de políticas de mix social aplicadas en muchos casos mundiales ponen en tela de juicio los supuestos beneficios de la gentrificación. En cualquier caso, pretender posicionamientos políticamente objetivos respecto a este tema es ya de por sí una quimera, su carácter conflictivo pareciera ser ya de naturaleza ontológica, y de por sí una invitación a discutir la enorme complejidad de aspectos de comunidad, desigualdad y transparencia en las políticas urbanas a escala de barrios (Wyly y Hammel, 2008).

Una limitación no menor del libro es su cierto eurocentrismo y norteamericanismo (resulta un tanto curioso que los tres casos de resistencia barrial a la gentrificación analizados en la última parte del libro provengan de Norteamérica), al defender también la tesis de una tendencia de expansión unidireccional (centro-periferia) de la gen- trificación. Esto sería observable tanto en el nivel de intervención de capitales financieros globales en mercados urbanos periféricos, como en la aparición de producción teórica desde estos últimos países, solo en el último tiempo. Sin embargo, el libro no pareciera abrirse a considerar que la diversidad de procesos de gentrificación en ciudades de las periferias mundiales, Latinoamérica incluida, podrían estar ocurriendo desde hace al menos dos décadas, con trayectorias de considerable mayor autonomía económica y política que las supuestas por estos autores.

Aunque ya no pareciera haber duda de que intensos desarrollos inmobiliarios y políticas de regeneración ocurren con gran intensidad en los centros y pericentros de las ciudades latinoamericanas, estos configuran efectos sociales y económicos a todas luces diferentes a aquellos descritos en el libro, por ejemplo: menor escala y capital de los mercados urbanos, desigual efectividad del sector público en balancear expansión y renovación urbanas, políticas impositivas particulares sobre el suelo (en algunos casos, como el chileno, extremadamente centralizadas), mayor disponibilidad de suelo periférico para la producción de vivienda (consecuentemente, menor presión sobre las áreas centrales y pericentrales) y, especialmente, abierta disimilitud en la prioridad que tiene la regeneración de zonas centrales en las agendas públicas.

En cualquier caso, Gentrification debiera ser una referencia sustancial para los estudios urbanos de ciudades latinoamericanas en los próximos años. Una invitación también, según plantean Lees, Slater y Wyly, a superar el cierto sesgo liberal dominante $y$ relativamente despolitizado de los estudios sobre gentrificación en el último tiempo.

\section{Referencias bibliográficas}

ATKINSON, R. \& BRIDGE, G. Gentrification in a global context: the new urban colonialism. Oxon: Routledge, 2005.

GARCÍA, L. Elitización: propuesta en español para el término Gentrificación. Biblio 3 W, Revista Bibliográfica de Geografía y Ciencias Sociales, 2001, vol. VI, No 332. 
Disponible en Internet: http://www.ub.es/ geocrit/b3w-332.htm

HAMNETT, C. Socio-economic change in London: professionalisation not polarization. Built Environment, 1994, vol. 31, № 3, p. 192-203.

LEY, D. Liberal Ideology and the postindustrial city. Annals of the Association of American Geographers, 1980, vol. 70, Nº 2, p. 238-258.

SLATER, T. The eviction of critical perspectives from gentrification research.
International Journal of Urban and Regional Research, 2006, vol. 30, No 4, p. 737-757.

SMITH, N. Toward a theory of gentrification a back to the city movement by capital, not people. Journal of the American Planning Association, 1979, vol. $45, N^{\circ} 4$, p. 538-548.

SMITH, N. The new urban frontier: gentrification and the revanchist city. New York: Routledge, 1996.

WYLY, E. \& HAMMEL, D. Commentary: urban policy frontiers. Urban Studies, 2008, vol. 45, No 12 , p. 2643-2648. 